\title{
The application of solar burst theory in the study of episodic jets
}

\author{
Zhiyu Yin ${ }^{1, \text { a }}$ \\ ${ }^{1}$ Penn State University, University Park 16802, USA; \\ ayinzhiyubj@126.com
}

Keywords: Solar burst theory; Episodic jets; Accretion disk

\begin{abstract}
In many astrophysics black hole accretion disk systems, the episodic jet formed by the plasma group is observed, but its origin is not yet understood. On the basis of the MHD model, this paper adds the relativistic effect, develops a new magneto hydrodynamic model to study the nature of the episodic jet, and draws the conclusion.
\end{abstract}

\section{Introduction}

Yuan et al [1] has made an important research on the episodic jets flow and obtained the certain achievement in 2009. In their model, mass ejection speed has reached relativistic velocities and observation also shows that episodic jets have a lot of Lorentz factor. But they didn't consider the theory of relativity, so their model has some limitations. Because of it, this paper in the base of Yuan et al episodic jets MHD model, considered the relativistic effect, developed a new model to study the generation and motion of episodic jets, to obtain more episodic jets physical information.

Because the outward flow in the black hole accretion disk system and the sun outward flow has similar forms and features in composition, and according to the current understanding of magnetic outward flow which under the environment of the sun, Yuan et al. established a magnetic fluid dynamics model to study black hole accretion disk system magnetic outward flow. But due to the environment of outward flow in the black hole accretion disk system and the environment of solar magnetic outward flow is very different, the outward flow velocity in the black hole accretion disk system close to the speed of light, the theory of relativity has an important influence on the evolution of the system which cannot be ignored. Therefore, in this work we used and improved the model of Yuan et al, joined the relativistic effects, rewritten the model equations, and developed a new magnetic fluid mechanics model to further study the intermediate episodic jets in the black hole accretion disk system.

\section{MHD model of episodic jets}

Taking the black hole at the center of the Milky way, Sgr A* and three solar masses of stellar mass black holes as example, this paper respectively study the Kinetic process of episodic jets in the supermassive black hole accretion disk system and low mass black hole accretion disk system, and compare the two different systems in the same process.

Due to the formula derivation process cumbersome and complex, the model used in the calculation formula of the specific derivation process is not given here, this paper simply list some of the major formulas, and all formula and the formula derivation process can reference the article content of the Lin and Forties [3], Yuan et al [2] ${ }^{[2]}$, and Meng et al [6].

A shear and turbulent motion occurs in the magnetic field in the Accretion Flow around the black hole, which leads to the formation of a magnetic flux rope in the magnetic field (see Fig 1). Before the occurrence of the current sheet, the expression of the potential field of the system $A_{p h}$ is:

$$
\begin{aligned}
& A_{C S}(z)=\frac{I_{0}}{C}\left[2 J \ln \left(\frac{z+\mathrm{i} h}{z-\mathrm{i} h}\right)+\mathrm{i} \operatorname{Ln}\left(\frac{z+\lambda}{z-\lambda}\right)\right] . \\
& z=x+y \mathrm{i}
\end{aligned}
$$




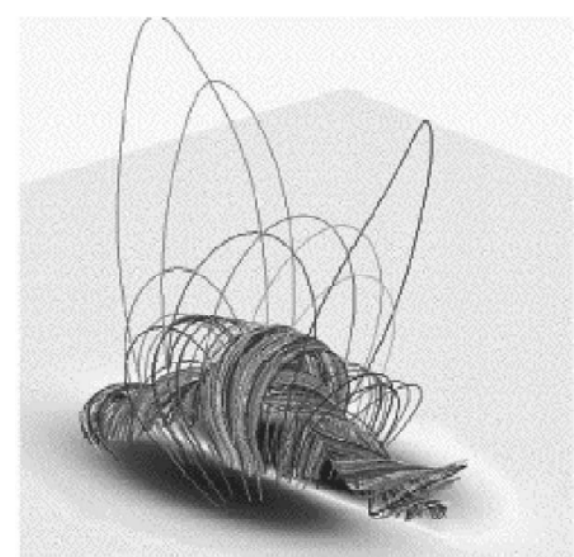

Fig. 1 The structure diagram of the magnetic flux rope

Using $B_{C s}(z)=-\partial A_{C S}(z) / \partial z$ relationship, we can get the expression of the magnetic field at the critical point of the system, as follows:

$$
B_{c s}(\mathrm{Z})=2 \mathrm{i} \frac{I_{0}}{c} \frac{h^{2} \lambda+\mathrm{z}^{2 \lambda}+2 h J\left(\mathrm{z}^{2}-\lambda^{2}\right)}{\left(\mathrm{h}^{2}+\mathrm{z}^{2}\right)\left(\mathrm{z}^{2}-\lambda^{2}\right)}
$$

This time the separatrix bubble as shown in Figure 2. It is connected to the closed region surrounded by a current sheet of the magnetic field lines from both ends, the region in the flux rope is ejected, is the so-called plasma blob, carried by the magnetic field energy formula:

$$
W_{m c}(\mathrm{z})=\frac{1}{8 \pi} \int B_{c s}{ }^{2}(\mathrm{z}) \mathrm{dV}=\frac{L}{8 \pi} \int B_{c s}{ }^{2}(\mathrm{z}) \mathrm{dS}
$$

In the above formula, $\mathrm{L}$ is the length of the magnetic flux rope, and $\mathrm{S}$ is the cross section area of the magnetic field, the expression of its boundary is $A_{c s}(\mathrm{z})=\mathrm{A}_{0}=I_{0} \pi / \mathrm{c}$.

Before the outbreak began, due to shear and turbulence of the black hole accretion disk accretion flow, magnetic energy in the magnetic flux rope gradual accumulation, when it reaches a threshold value, the system will lose the original dynamic balance, and quickly release the accumulation of energy in the form of catastrophe. In this process, the flux ropes were ejected outward, and formed a group of plasma blob of episodic jets. During the outward motion of the plasma blob, the magnetic reconnection flux magnetic flux from the surrounding background magnetic field into the plasma blob. When the plasma blob mass ejection from accretion flow, the energy it carries is twice about the energy in the outbreak of the critical point, namely:

$$
W_{m p}=2 W_{m c}
$$

In this process, the flux ropes were ejected outward, and formed a group of plasma blob of episodic jets. The motion of this plasma blob is governed by the following equations:

$$
m \gamma^{3} \frac{d^{2} h}{d t^{2}}=\frac{1}{c}\left|I \times B_{e x t}\right|_{h}-F_{9}
$$

Where $\mathrm{m}$ is the quality of the plasma mass per unit length and $\gamma=1 / \sqrt{1-v^{2} / c^{2}}$ is the Lorentz factor, $\mathrm{h}$ is the surface distance between plasma blob and accretion disc, $\mathrm{f}$ is the gravity, $\mathrm{I}$ is current of the magnetic flux rope, and B is except $\mathrm{I}$, the sum of the magnetic field from the accretion disk, the corona and current sheet. We take $(y=0)$ as the equatorial plane, $(x=0)$ as the axis of the accretion flow, and establish the (x,y) coordinate system (reference Figure 3 ). The model assumes that the initial location of the flux rope is $(\mathrm{x}, \mathrm{y})=\left(5 \mathrm{r}_{g}, 10 \mathrm{r}_{g}\right), \mathrm{r}_{g}=2 G M / c^{2}$ is the Schwarzschild radius of the black hole. This initial position is far from the center of the black hole, so we only consider the theory of special relativity in the model without considering the role of general relativity. 


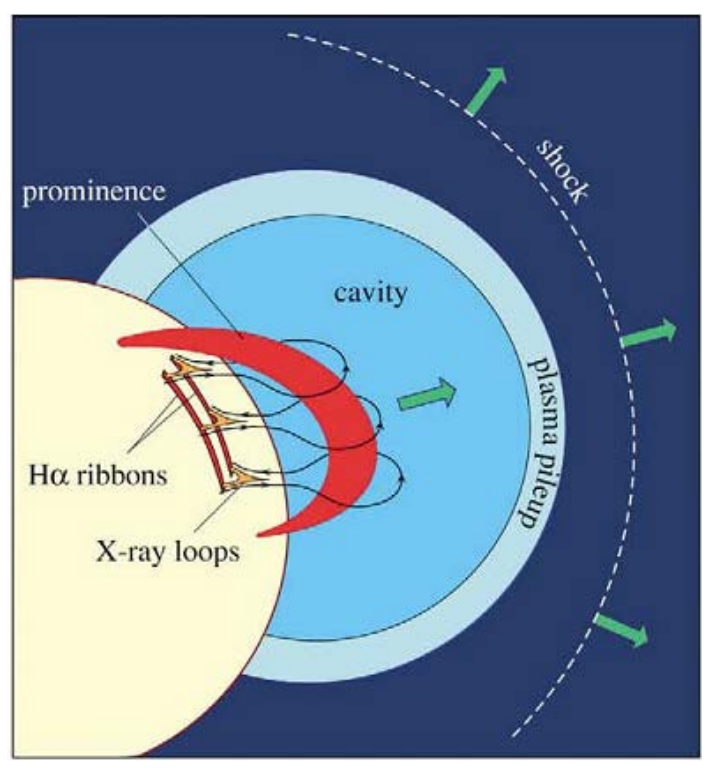

Fig. 2 The magnetic structure of flare eruptive prominence in CME process When Plasma mass move, the rate of change of kinetic energy with time is

$$
\begin{aligned}
& \frac{d W_{K E}}{d t}=\frac{d\left[\mathrm{~m}(\gamma-1) \mathrm{c}^{2}\right]}{d t} \\
= & m \gamma^{3} h \frac{d^{2} h}{d t^{2}} \\
= & \frac{I B_{e x t} h}{c}-\frac{G M \gamma m}{\left(H_{0}+h\right)^{2}} \dot{ } \\
= & \frac{B_{0}{ }^{2} \lambda^{4}}{8 h L_{P Q}{ }^{2}}\left[\frac{H_{P Q}{ }^{2}}{2 h^{2}}-\frac{\left(\mathrm{p}^{2}+\lambda^{2}\right)\left(\mathrm{h}^{2}-\mathrm{q}^{2}\right)+\left(\mathrm{q}^{2}+\lambda^{2}\right)\left(\mathrm{h}^{2}-p^{2}\right)}{h^{2}+\lambda^{2}}\right] h-\frac{G M \gamma m}{\left(H_{0}+h\right)^{2}} h \\
& H_{P Q}=\sqrt{\left(\mathrm{h}^{2}-\mathrm{p}^{2}\right)\left(\mathrm{h}^{2}-\mathrm{q}^{2}\right)}, L_{P Q}=\sqrt{\left(\lambda^{2}+\mathrm{p}^{2}\right)\left(\lambda^{2}+q^{2}\right)}, G=6.67 \times 10^{8} \mathrm{~cm}^{3} \mathrm{~g}^{-1} \mathrm{~s}^{-2}
\end{aligned}
$$

constant, $\mathrm{M}$ represents the quality of the black hole.

As highly ionized plasma was frozen in magnetic field, the plasma is bound in the vicinity of the magnetic structure. A large number of plasma blob with magnetic flux in the crown layer, magnetic reconnection by current sheet into the ejections from the background environment. At this time, in the system of Alfven speed is

$$
V_{A}=\left|B_{y}\left(0^{ \pm}, \mathrm{y}_{0}\right)\right| / \sqrt{4 \pi \rho\left(\mathrm{y}_{0}\right)}
$$

Therefore, the rate of change of the quality of projectiles with time can be described as ${ }^{[5]}$ :

$$
\frac{d m}{d t}=B_{0} M_{A} \sqrt{\frac{\rho_{0}}{\pi}} \frac{\lambda^{2}(\mathrm{q}-\mathrm{p})\left(\mathrm{h}^{2}+\lambda^{2}\right)}{\left(\mathrm{h}^{2}-y_{0}{ }^{2}\right)\left(y_{0}{ }^{2}+\lambda^{2}\right)} \times \sqrt{\frac{f\left(\mathrm{y}_{0}\right)\left(\mathrm{q}^{2}-y_{0}{ }^{2}\right)\left(y_{0}^{2}-p^{2}\right)}{\left(\lambda^{2}+p^{2}\right)\left(\lambda^{2}+\mathrm{q}^{2}\right)}}
$$

In the formula, $\mathrm{f}(\mathrm{y})$ is a dimensionless plasma density distribution function of coronal layer, with mass density distribution of $\rho(\mathrm{y}) . \rho(\mathrm{y})=\rho_{0} f(\mathrm{y}) . \rho_{0}$ is the mass density of the accretion disk surface. Because there is no generally accepted model can explain the density distribution of magnetic disk in the crown. We refer to the model of Yuan et al, using density function of sun model to examine the distribution of the accretion disk corona layer material distribution. Therefore, $\mathrm{f}(\mathrm{y})$ uses the following expressions:

$$
\begin{aligned}
& f(\mathrm{y})=a_{1} \mathrm{z}^{2}(\mathrm{y}) \mathrm{e}^{a_{2} z(\mathrm{y})}\left[1+a_{3} \mathrm{z}(\mathrm{y})+a_{4} \mathrm{z}^{2}(\mathrm{y})+a_{5} \mathrm{z}^{3}(\mathrm{y})\right] \\
& \mathrm{z}(\mathrm{y})=\lambda /(\lambda+y), a_{1}=0.001272, a_{2}=4.8039, a_{3}=0.29696, a_{4}=-7.1743, a_{5}=12.321
\end{aligned}
$$


When $\mathrm{y}=0, \mathrm{f}(0)=1$.

The equation (6) and (7) and Meng et al [6]. Article (24) - (26) simultaneous into equations, solute the equations to get the description of the dynamic process of the system in the wake of the catastrophic loss of balance.

\section{Motion process of plasma blob}

Based on the results of the center of the Milky Way Galaxy high quality black hole $A *$ Sgr in the accretion flow and the corona and GRB environment constant star black hole research work, in this work we study the movement of episodic jets. In our work, assuming the crown layer of Black Hole Accretion Disc is weak magnetic field and a leading order approximation. The magnetic reconnection rate used in the calculation is 0.01 . In the model, the distance between the two points is $2 \lambda_{0}, \lambda_{0}=5 r_{g}$, and the length of the magnetic flux rope is $L=2 \lambda_{0}$. These model parameters are selected according to the observation results.

According to the values of parameters in the model of Yuan et al, in the process of calculation, we select the mass of the unit length plasma blob, respectively $m_{1}=4.54 \times 10^{3} \mathrm{gcm}^{-1}$, $m_{2}=4.54 \times 10^{4} \mathrm{gcm}^{-1}$. The surface magnetic field intensity of the accretion disk of Sgr A* is $B_{0}=2 I_{0} /\left(c \lambda_{0}\right)=16 \mathrm{G}$, The Schwarzschild radius of the black hole is $5.9 \times 1011 \mathrm{~cm}$. By solving the equations of this paper presented earlier, we can get the dynamic characteristics of Sgr A* Black Hole Accretion Flow ejection plasma blob. The calculation of the results is given in the form of the equation, and lighter plasma blob are marked by a solid line, and heavier plasma blob are marked by dotted lines. Our computational results show that from the black hole accretion disk ejection of the plasma blob easily are accelerated to relativistic speeds, and fast access to large Lorentz factor, as shown in Figure 3, this point and observation results consistent. Small mass of the plasma blob is more likely to be accelerated to relativistic velocity than big mass of the plasma blob.

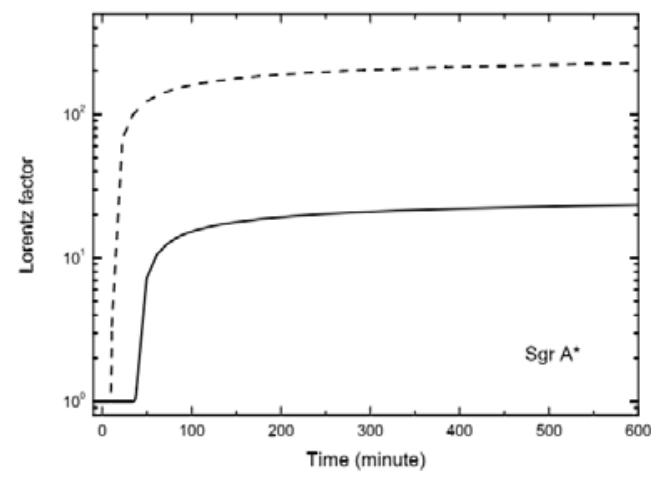

Fig. 3 Change of Lorentz factor in Plasma clusters of A* Sgr episodic jets with time

Figure 4 shows the changes in the cross-sectional area of the projectile plasma blob with time. Because the length of the plasma blob is a fixed parameter, the change of the transverse section area of the plasma blob can reflect the change of the volume of the plasma blob body during the escape process. From Figure 4 that plasma blob ejection group in the movement process is expanding, first experienced a rapid expansion and expansion process slows down. Under the same conditions, small mass of the plasma blob expands faster. 


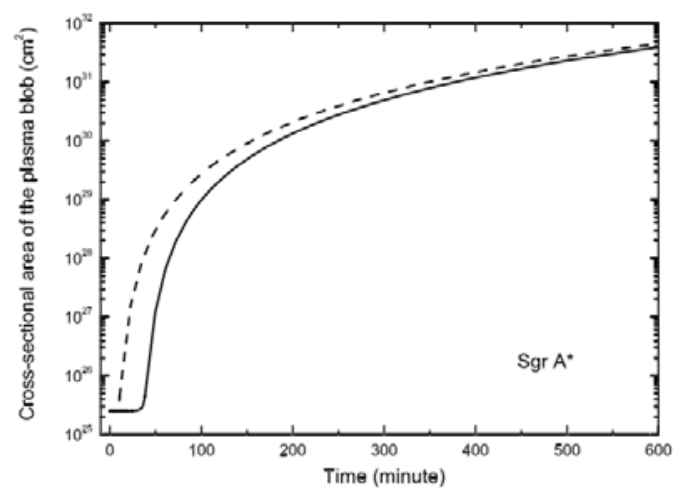

Fig 4 The change of cross sectional area of the plasma blob of A* Sgr episodic jets flow with time.

The change of the mass of the plasma blob with time is given in figure 5. Although magnetic reconnection will make some plasma in coronal layer eject into the plasma blob by current sheet, but the mass of plasma blob in plasma ejection process remains the same. Because the plasma in corona layer is very thin and density is very low, compared to the plasma blob original, the mass of plasma with the magnetic reconnection eject in can be neglected.

With the same method, we can also calculate the dynamic process of episodic jets in the middle of the accretion disk system of the constant star black hole. For the stellar black hole, the mass of the unit length plasma mass is $\mathrm{m} 1=6.15 \times 1019 \mathrm{gcm}^{-1}, \mathrm{~m} 2=6.15 \times 1020 \mathrm{gcm}^{-1}$ The surface magnetic field intensity of the accretion disk is $\mathrm{B} 0 \approx 1015 \mathrm{G}$ (reference to calculation results of Zhang and Yuan's model). The Schwarzschild radius for stellar black holes is $r_{g}=8.85 \times 105 \mathrm{~cm}$.

From Figure 5, we can find that both the episodic jets generated by the accretion disk of the stellar black hole and generated by the massive black hole has experience the same process of accelerate, and in this process, the expansion and quality of the roughly maintained unchanged. But due to stellar mass black holes has strong magnetic field, the onset process is very intense and can more quickly ejected plasma blob as released enormous energy. So, compared to episodic jets generated by massive black holes, the time that the episodic jets generated by the stellar black hole accretion disk accelerated to relativistic velocity is shorter, and can obtain greater Lorentz factor.

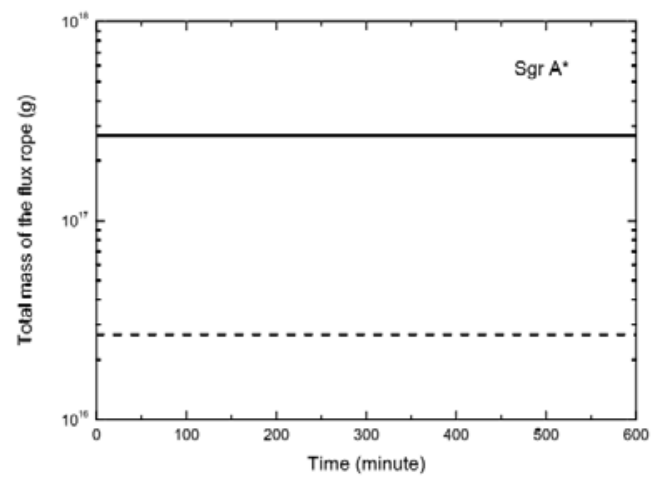

Fig. 5 The change of the mass of the plasma blob of A* Sgr episodic jets flow with time.

\section{Conclusion}

In many astrophysics black hole accretion disk systems, the episodic jet formed by the plasma group is observed, but its origin is not yet understood. On the basis of the MHD model, this paper adds the relativistic effect, develops a new magneto hydrodynamic model to study the nature of the episodic jet.

Taking the A* Sgr system and the central engine of the central engine of the massive black hole in the galaxy as an example, we use the new model to study the motion of the plasma blob.

In this two examples, our calculations show that plasma blob can quickly obtain high Lorentz factor; they are outward eject at relativistic speed; the total mass of the plasma blob in the 
movement process remained unchanged; plasma blob carrying huge energy away from the black hole accretion disk system, the size of the magnetic energy mainly determined by the Accretion Disc surface of magnetic field intensity, the stronger the magnetic field, the larger the energy.

\section{Reference}

[1] Yuan, F., \&Zhang, B. 2012, ApJ, 757, 56 Episodic Jets As The Central Engine Of Gamma-Ray Bursts.

[2] Yuan, F, Lin, J., Wu, K., \&Ho, L. C. 2009, MNRAS, 395, 2183 A Magnet hydro dynamical Model for the Formation of Episodic Jets.

[3] Lin, J., \&Forbes, T. G., 2000, JGR, 105, 2375 Effects of reconnection on the coronal mass ejection process.

[4] Priest, E. R., 1982, Dordrecht, Holland; Boston: D. Reidel Pub. Co Hingham, Solar magneto-hydrodynamics.

[5] Lin, J., Mancuso, S., \&Vourlidas, A. 2006, ApJ, 649, 1110 Theoretical Investigation of the Onsets of Type || Radio Bursts during Solar Eruptions.

[6] Meng, Y., Lin, J., Zhang, L., Reeves, K. K.,2hang, Q. S., \&Yuan, F 2014, ApJ, 785,62 An MHD Model For Magnetar Giant Flares.

[7] Amari, T., Luciani, Mikic, Z., Linker,J., 2000, ApJ, 529, L49 A $\quad$ Twisted Flux Rope for Coronal Mass Ejections and Two-Ribbon Flares.

[8] De Villiers, J. P., Hawley, J.F., \&Krolik, J. H. 2003, ApJ, 599, 1238 Magnetically Driven Accretion Flows in the Kerr Metric. I. Models and Overall Structure.

[9] Yuan, F., Quataert, E.,\&Narayan, R. 2003, ApJ, 598, 301 Nonthermal Electrons in Radiatively Inefficient Accretion Flow Models of Sagittarius A*. 